\title{
Interactive comment on "New strategies for vertical transport in chemistry-transport models: application to the case of the Mount Etna eruption on March 18, 2012 with CHIMERE v2017r4" by Mathieu Lachatre et al.
}

\section{Anonymous Referee \#2}

Received and published: 20 May 2020

\section{I - General comments}

This manuscript presents new numerical modeling approaches to represent vertical transport of pollutants plumes in the upper troposphere with Eulerian ChemistryTransport Models (CTM). The aim is to limit the excessive vertical diffusion of the plumes of pollutant in this kind of numerical representation.

Different numerical strategies are considered to address this issue : different vertical wind diagnosis, different advection scheme and different vertical resolution. The sen-

Printer-friendly version

Discussion paper 
sitivity of the simulation of a plume transport event to the different numerical choices considered in this work is evaluated on the case of the Mount Etna's eruption of March 18, 2012.

Its a topic of scientific interest and certainly within the scope of Geoscientific Model Development. The general presentation of the work is logically and clearly organized. However the added value of this work could be improved with the clarification and/or the development of some results.

II - Specific Comments

\section{Section 2.1}

p4 17 and 113: Could the authors precise which CHIMERE version has been used? 2013, 2017 or 2016 ?

p4 I14 : The horizontal resolution of the WRF simulation should be mentioned.

p4 117 : The authors should provide the limits of the vertical layers (at least in supplement with figure S4).

p4 116-17: There is an in-depth discussion on the relationship between horizontal resolution and vertical resolution in the article by Zhuang et al. (2018) that the authors cite in the introduction, but nothing is said here on this subject. Beyond this point, it is weird to see that the chosen vertical extension of the domain does not provide any possibility to reproduce the highest part of the plume as seen by the observations (cf. figure $2 \mathrm{e}, \mathrm{S} 3$ and S4). All the more so when we see that the meteorological simulation would allow the domain to be extended. Could the authors explain how they chose the different vertical resolutions tested?

p4 I17 : These different vertical resolutions rely on an oversampling of the same simulated meteorological fields. Could we expect to get significantly different vertical profile with a meteorological simulation carried out with a finer vertical grid?

Printer-friendly version

Discussion paper 
p4 117 : Only part of the 33 vertical levels of the meteorological grid is used for the interpolation on the dispersion grid. The number of levels concerned could be specified in this paragraph.

p10 I11 and p12 15: The comparison between the different vertical resolutions involve an aspect which may deserve a bit more detailed discussion. Which kind of boundary conditions are applied for the pollutant concentrations? With a plume injection in the last layer of the model (at least when 20 levels are used) it seems that the boundary conditions could play a role. What happens when the flux is downward oriented? (here again the choice of a larger vertical extension would be more relevant).

p13 I1-4: Could the authors provide the levels concerned in these tests?

p13 15-7 : The authors mention an "injection to a unique altitude". It implies the different simulations with the different vertical resolution do not start with the same vertical extension of the plume. It would be interesting to isolate the impact of this initial discrepancy that cannot be associated to an excessive diffusion of the advection scheme. I guess this could be done with a 50 or 99 levels simulations run with an injection uniformly distributed over the different layers corresponding to the injection layer of the 20 levels simulation.

p14 section 3.3 : With the location of "the model column with the strongest vertically integrated SO2 content" the authors have chosen a very aggregated indicator for the comparison between satellite soundings and model results. I assume this choice was made for sake of simplicity in the presentation of the results. However, seeing that the configuration option can lead to some plume splitting, it would be interesting to have more information concerning the horizontal extension of the plume in the different cases.

Printer-friendly version

p14 I23 : It is not clear to me if the results in figure 5 present average over different configuration options. For instance in the first panel, the simulations with the different advection scheme are compared. Do the number are averages over the different

Discussion paper

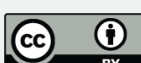


vertical resolutions (the vertical resolution is not mentioned either in the text or in the label of the figure)? Does this imply that there is few interaction between the tested options? From figure 4 we can see that the WRFW-DL-99 simulation is not the closest to the observation at the final stage. This may not be the intuition get from the results presented.

p16 I18-20 : Could the authors precise how the distribution are built? It is not clear for me if it represents different time steps, different locations, or a mix? Are the observations uncertainties are represented in this figure?

p17 16-8 : These lines are frustrating from my point of view. The authors focus their work on the excessive vertical diffusion in the dispersion model and the only comparison of the model results to observation concerning the plume vertical extension indicates that this plume property is underestimated. Could the authors provide a more in-depth discussion concerning this point? Some considerations concerning the time evolution of the maximum concentration (modeled and observed) could be useful here to convince the readers that a less diffusive treatment of the advection is really suitable. Since the transport in this application is linear, even a normalized comparison to the "initial" ( sounding number 1 ) maximum concentration would be useful.

III - Technical corrections

p5 I11: It seems an "overbar" is missing for notation consistency.

p11 label table 3 : The last sentence should probably be in the label of Figure 2.

p18 121 : erroneous citation

Interactive comment on Geosci. Model Dev. Discuss., https://doi.org/10.5194/gmd-2020-62, 2020.

Printer-friendly version

Discussion paper 